\title{
ARTICLE
}

\section{Transcriptome profiling of Cucumis melo fruit development and ripening}

\author{
Hong Zhang ${ }^{1,3}$, Huaisong Wang ${ }^{2,3}$, Hongping $\mathrm{Yi}^{1}$, Wenqiang Zhai ${ }^{1}$, Guangzhi Wang ${ }^{1}$ and Qiushi Fu ${ }^{2}$
}

\begin{abstract}
Hami melon (Cucumis melo) is the most important melon crop grown in the north-western provinces of China. In order to elucidate the genetic and molecular basis of developmental changes related to size, flesh, sugar and sour content, we performed a transcriptome profiling of its fruit development. Over 155000000 clean reads were mapped to MELONOMICS genome, yielding a total of 23299 expressed genes. Of these, 554 genes were specifically expressed in flowers, and 3260 genes in fruit flesh tissues. The 7892 differentially expressed genes (DEGs) were related to fruit development and mediated diverse metabolic processes and pathways; 83 DEGs and 13 DEGs were possibly associated with sucrose and citric acid accumulation, respectively. The quantitative real-time PCR results showed that six out of eight selected candidate genes displayed expression trends similar to our DEGs. This study profiled the gene expression related to different growing stages of flower and fruit at the whole transcriptome level to provide an insight into the regulatory mechanism underlying Hami melon fruit development.
\end{abstract}

Horticulture Research (2016) 3, 16014; doi:10.1038/hortres.2016.14; Published online 27 April 2016

\section{INTRODUCTION}

Melon (Cucumis melo L.) is an important annual diploid plant belonging to the Cucurbitaceae family. Melon plants produce large edible fruits that serve as an important dietary component worldwide. ${ }^{1}$ With increasing interest in biological properties and economic importance, melon has become an attractive model for studying valuable features, such as fruit ripening, ${ }^{2}$ sex determination $^{3,4}$ and phloem physiology. ${ }^{5}$

The fruit development of medium maturing melon mainly includes four typical stages: young fruit, expanding, premature and mature. The open female flowers are ready for pollination only for about a day. Therefore, hand pollination is generally performed on the same day as flower opening. After pollination, cell division in the ovary is increased. Ten days after flowering (pollination; $10 \mathrm{DAF}$ ), ovarian epidermal cells stop proliferating leading to young fruit stage, with a small volume. Subsequently, following a rapid increase in the volume of young fruit, the cell diameters of the mesocarp, endocarp and placenta increase quickly resulting in fruit enlargement. Twenty days after flowering (20 DAF), the melon fruit enters the expanding stage, with the maximum fruit volume, and sugar content at a very low level. Subsequently, the fruit length is continuously increased, with rapidly increased sucrose content. Thirty days after flowering (30 DAF), the melon fruit enters a premature stage, cell expansion stops with maximum fruit volume and flesh firmness. The sucrose content consistently increases and reaches the maximum (60\% of total sugar), with flesh turning soft in texture. Forty days after flowering (40 DAF), the melon fruit is considered fully mature or developed. At this stage, the sucrose content reaches the highest level, along with other nutritional components.

In recent years, a series of -omics studies on melon have been conducted. Dahmani-Mardas et al. ${ }^{6}$ compiled a list of candidate genes and isolated key mutants with enhanced fruit quality.
Garcia-Mas et al. ${ }^{7}$ used the 454 pyrosequencing method to de novo sequence the melon double-haploid line DHL92 and obtained $375 \mathrm{Mb}$ of assembled genome. In the same study, the melon genome sequence was phylogenetically compared with the genomes of cucumber and other plant species. Corbacho et $a l^{8}{ }^{8}$ used pyrosequencing to identify the candidate genes involved in the early induction of mature melon fruit abscission. Furthermore, the international cucurbit genomics initiative (ICuGl; http://www.icugi.org) conducted several genome-wide studies ${ }^{7-9}$ and provided valuable data to understand the mechanisms underlying sugar metabolism and mature fruit abscission in climacteric melon.

Hami melon is a melon cultivar geographically distributed in Xinjiang Turpan Hami area. As an ecological type, it mainly includes C. melo var chandalak, ameri, cassaba and inodorus cultivars. Hami melon is highly popular worldwide for its distinct flavor. 'Flavor No. 4' is a new cultivated medium maturing variety of Hami melon with unique sweet and sour taste. In order to better understand the processes of sucrose and organic acid accumulation in flesh, we performed a comprehensive transcriptome profiling of fruit flesh of 'Flavor No. 4'. Our study may shed new light on the dynamics and differential expression of genes during fruit development and ripening of Hami melon.

\section{MATERIALS AND METHODS}

Plant materials

Melon plants (C. melo var ameri, cv 'Flavor No. 4') were grown in the greenhouse at the Institute of Vegetables and Flowers, Chinese Academy of Agricultural Sciences (Beijing, China) in 2013. Flowers were tagged at anthesis and one fruit was allowed to develop per plant. When melon flowers opened, the female flowers were hand-pollinated immediately. The

\footnotetext{
${ }^{1}$ Hami Melon Research Center, Xinjiang Academy of Agricultural Science, Urumqi 830091, China and ${ }^{2}$ The Department of Cucurbitaceae Vegetables Genetics and Breeding, Institute of Vegetables and Flowers of the Chinese Academy of Agricultural Sciences, Beijing 100081, China.

${ }^{3}$ These authors contributed equally to this work.

Correspondence: H Zhang (hzhang0625@163.com)

Received: 30 December 2015; Revised: 13 March 2016; Accepted: 14 March 2016
} 
fruits were collected 10, 20, 30 and 40 DAF. Female flower is the reproductive organ producing melon fruit and seeds. In order to comprehensively understand the process of Hami melon fruit development, the fully opened female flowers (0 DAF) were collected and included in this study. At each time point, three fruits/flowers were harvested from three different plants. Flesh mesocarp was obtained from the centerequatorial portion of each fruit. Tissue pieces were immediately frozen in liquid nitrogen and stored at $-80^{\circ} \mathrm{C}$ for the following analysis.

High-performance liquid chromatography of sugar and organic acid content

The content of sugars and organic acids in fruit flesh were analyzed by high-performance liquid chromatography as previously described. ${ }^{10}$

\section{RNA isolation}

Total RNA was extracted from each tissue using TRIzol kit (Invitrogen, Carlsbad, CA, USA) according to the manufacturer's instructions. Subsequently, the RNA samples were treated with RQ1 DNase (Promega, Madison, WI, USA) to remove both double- and single-stranded DNA. The quality and quantity of the purified RNA were determined by measuring the absorbance at $260 \mathrm{~nm} / 280 \mathrm{~nm}$ (A260/A280) on Smart Spec Plus Spectrophotometer (Bio-Rad, Philadelphia, PA, USA). RNA integrity was further verified by $1.5 \%$ agarose gel electrophoresis.

\section{Complementary DNA library preparation}

For each sample, $10 \mathrm{mg}$ of total RNA was used for RNA sequencing (RNAseq) library preparation. Polyadenylated mRNAs were purified and concentrated with magnetic beads oligo (dT) (Invitrogen) before directional RNA-seq library preparation. Purified mRNAs were fragmented at $95^{\circ} \mathrm{C}$ followed by end-repair and $5^{\prime}$ adapter ligation. The reverse transcription was performed with reverse transcription primer harboring 3 ' adapter sequence and randomized hexamer. The complementary DNAs were purified and amplified and PCR products corresponding to 200$500 \mathrm{bp}$ were purified, quantified and stored at $-80^{\circ} \mathrm{C}$ until used for sequencing.

\section{Whole-transcriptome sequencing}

For high-throughput sequencing, the prepared libraries were subjected to Illumina Hiseq 2000 system 100 nucleotides (nt) pair-end sequencing (ABlife, Inc., Wuhan, China).

\section{RNA-seq raw data clean and alignment statistics}

First, raw reads containing more than $2-\mathrm{N}$ bases were discarded, and the remaining reads were processed by clipping adapters. Low-quality bases were removed. Reads less than 16 nt were also dropped. FASTX-Toolkit (Version 0.0 .13 ) was used to obtain the clean reads. Subsequently, clean reads were aligned to MELONOMICS genome sequence (https://melono mics.net/) by Tophat $2 .{ }^{11}$ On the basis of the genome location of the reads, aligned reads containing more than one genome location were discarded due to their ambiguous location. Uniquely localized reads were used to calculate the read number and RPKM (reads per kilobase and per million) value for each gene, according to their genome location. Other statistical results, such as gene coverage and depth, read distribution around start codon and stop codon, were also obtained. RNA-seq data were deposited in the NCBI Sequence Read Archive (NCBI SRA SRX641227).

\section{Hierarchical clustering analysis}

Hierarchical clustering was performed to calculate the cluster of genes set by Cluster 3.0 software. Heat maps showing expression profiles $\left(\log _{2}\right.$ RPKM) were generated using Java Tree View (http://jtreeview.sourceforge. net, Baryshnikova Lab, Princeton University, Princeton, NY, USA).

Data analysis of transcriptome profiles of differentially expressed genes

Differentially expressed genes (DEGs) between two samples were analyzed using edge $\mathrm{R}_{1}{ }^{12}$ one of $\mathrm{R}$ packages. For each gene, the $P$-value $<0.01$ and foldchange $>2$ or $<0.5$ were considered as the significant threshold. All DEGs were mapped to terms in Gene Ontology (GO) and Kyoto Encyclopedia of Genes and Genomes (KEGG) databases. For GO analysis,
Hami melon-expressed genes were aligned to the NR database with a $1 \mathrm{e}^{-5}$ $E$-value threshold, and the alignment were assigned GO functional descriptions by Blast2GO software. ${ }^{13}$ For KEGG analysis, Fisher's exact test was used to define the enrichment of each GO term and KEGG pathway. Other statistical results were obtained using $\mathrm{R}$ software.

\section{Quantitative real-time PCR analysis}

To validate the accuracy of our transcriptome profiling, the gene expression of eight randomly selected candidate genes was evaluated by quantitative real-time PCR. Total RNA was extracted from female flowers at $0 \mathrm{DAF}$ and fruit flesh at $10 \mathrm{DAF}, 20 \mathrm{DAF}, 30 \mathrm{DAF}$ and $40 \mathrm{DAF}$ with TRIzol kit (Invitrogen). The total RNA was reverse transcribed using QuantiTect Reverse Transcription kit (Qiagen, Valencia, CA, USA) according to the manufacturer's protocol. Actin was used as an internal control. Real-time monitoring of PCR was performed with ABI 7500 Real Time System and SYBR GREEN PCR Master Mix (Applied Biosystems, Grand Island, NY, USA). Reactions were performed at $94^{\circ} \mathrm{C}$ for $2 \mathrm{~min}$, and then cycled at $95^{\circ} \mathrm{C}$ for $30 \mathrm{~s}, 48-54{ }^{\circ} \mathrm{C}$ for $30 \mathrm{~s}, 72{ }^{\circ} \mathrm{C}$ for $30 \mathrm{~s}$ for 35 cycles and then $72{ }^{\circ} \mathrm{C}$ for $10 \mathrm{~min}$. Each assay was performed in triplicates. Data analysis was performed using the $2^{-\Delta \Delta C t}$ method described previously. ${ }^{14}$

\section{RESULTS}

Fruit size, sugar and organic acid content of 'Flavor No. 4' during fruit development

'Flavor No. 4', a medium maturing variety of Hami melon, was used in the present study. It belongs to the C. melo var ameri (pang) Greb. It is a Hami melon cultivar that produces short oval shape, medium size and yellow skin melon with 10 green longitudinal grooves on the surface. This type of melon contains white juicy flesh with unique sweet and sour taste (Figures $1 \mathrm{a}, \mathrm{c}$ and d). The fruit size, sugar and organic acid content of fruit flesh at four typical developmental stages were examined. At 10 DAF, the volume of melon fruit was still small; from 10 to $20 \mathrm{DAF}$, the fruit diameter and length both increased rapidly; from 20 to 30 $D A F$, the fruit diameter slightly increased, the fruit length continuously rapidly increased; from 30 to $40 \mathrm{DAF}$, the fruit volume increased to the maximum (Figures $1 \mathrm{a}$ and $\mathrm{b}$ ). The sucrose, glucose and fructose contents in the fruit flesh were examined by high-performance liquid chromatography. As shown in Figure 1c, the glucose and fructose content remained at a low level during fruit development, whereas the sucrose content increased markedly from 20 to $40 \mathrm{DAF}$, reaching its highest level at $40 \mathrm{DAF}$. This finding suggested that rapid sucrose accumulation at the medium and late developmental stages contributed to the sweet taste of fruit flesh. The contents of citric acid (CA) and malic acid were detected by high-performance liquid chromatography. As shown in Figure 1d, the content of CA increased quickly from 20 to $40 \mathrm{DAF}$, and the CA content during 20-30 DAF increased even faster than that at 30-40 DAF. However, the malic acid level decreased slightly during fruit development indicating that the rapid accumulation of $C A$ in the medium and late developmental stages determined the sour flavor of fruit flesh.

Transcriptome profiles of the female opening flowers and the flesh mesocarp

RNA-seq was carried out on the complementary DNA libraries derived from the female opening flowers (0 DAF) and the flesh mesocarp of fruit at 10,20, 30 and 40 DAF. The processed clean reads were aligned to MELONOMICS genome sequence. An overview of the results is displayed in Table 1. The majority of reads from each sample (76.07-94.48\%) were successfully aligned, except that the alignment rate for sample 10 DAF was relatively low (52.19\%). We found the reads from sample 10 DAF contained many 20-40 nt short reads, which cannot be mapped to the reference genome. Between 12 and 24 million reads per sample were uniquely aligned to the MELONOMICS genome and over 18000 expressed genes were detected in each sample (Table 1). 
a

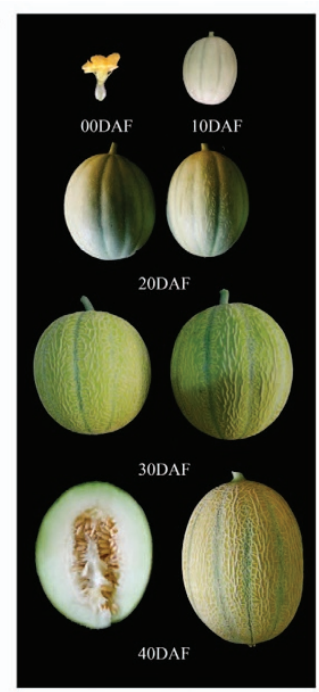

C

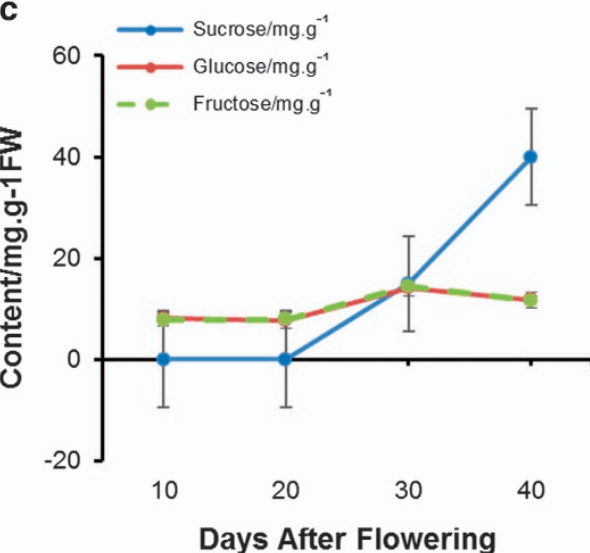

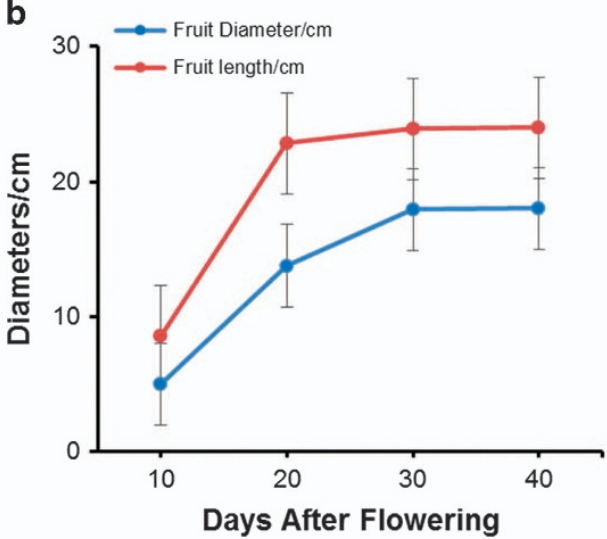

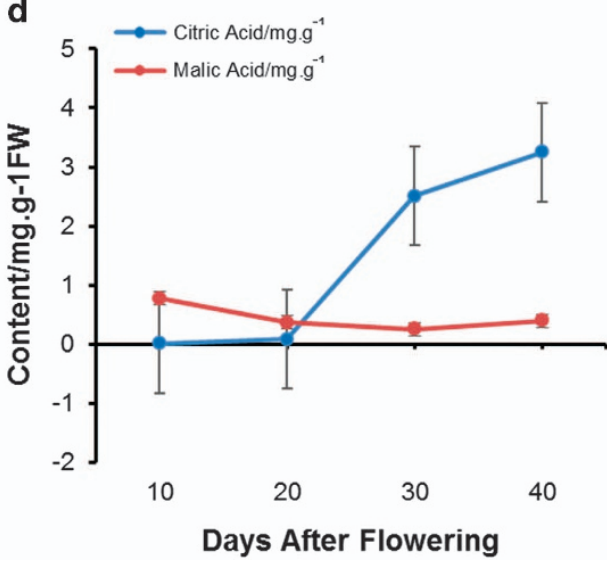

Figure 1. (a) The opening female flower and melon fruit of 'Flavor No. 4' at different developmental stages. (b) The size (length, diameter) of 'Flavor No. 4' fruit at 10, 20, 30 and 40 days after flowering (DAF). Data are the means of three measurements and s.e. values. (c) Soluble sugars (sucrose, glucose and fructose) concentrated in 'Flavor No. 4' fruit flesh at 10, 20, 30 and 40 DAF. Data represent the means of three extractions and s.e. values. (d) Organic acid (citric acid and malic acid) concentration in 'Flavor No. 4' fruit flesh at 10, 20, 30 and 40 DAF. Data represent the means of three extractions and s.e. values.

Table 1. Summary of RNA-seq data collected at five important stages of fruit development

\begin{tabular}{lccccc}
\hline Category & O DAF & 10 DAF & 20 DAF & 30 DAF \\
\hline Input reads & 25310332 & 25757533 & 20324207 & 24080391 & 40 DAF \\
Aligned to genome & $23735470(93.78 \%)$ & $13442267(52.19 \%)$ & $15459847(76.07 \%)$ & $22746535(94.48 \%)$ & $18036713(91.27 \%)$ \\
Uniquely aligned to genome & $23449265(92.65 \%)$ & $12683194(49.24 \%)$ & $15215812(74.87 \%)$ & $22509389(93.48 \%)$ & $17814425(90.14 \%)$ \\
Detected genes & 20366 & 21447 & 19815 & 18468 & 18484 \\
\hline
\end{tabular}

Abbreviations: DAF, days after flowering; RNA-seq, RNA sequencing. Read counts in the middle section are expressed in numbers (left) or as a percentage of the input reads processed (right) for each sample.

On the whole, 23299 of the 27427 annotated genes (84.95\%) were detected in flower and flesh mesocarp samples.

We found that 20206 and 22922 genes were expressed in female flowers (0 DAF) and fruit flesh (10-40 DAF), respectively, with 554 genes specifically expressed in 0 DAF (Figure 2), and 3260 genes specifically expressed in fruit at 10-40 DAF (Figure 2). Hami melon-expressed genes were assigned to GO functional descriptions based on their sequence matches to known proteins in the NR database. Hami melon-expressed genes were aligned to the NR database with a $1 \mathrm{e}^{-5} E$-value threshold, and the alignment were assigned GO functional descriptions by Blast2GO software. ${ }^{13}$ In female flowers, 2822 genes were assigned with at least one GO term in the biological process category, 2890 genes in the molecular function category and 1759 in the cellular component category. Although in fruit flesh, 2960 genes were assigned with at least one GO term in the biological process category, 3043 genes in the molecular function category and 1841 in the cellular component category. Cellular process, cell part and binding are the most abundant $\mathrm{GO}$ slims within the biological process, cellular component and molecular function categories, respectively. 


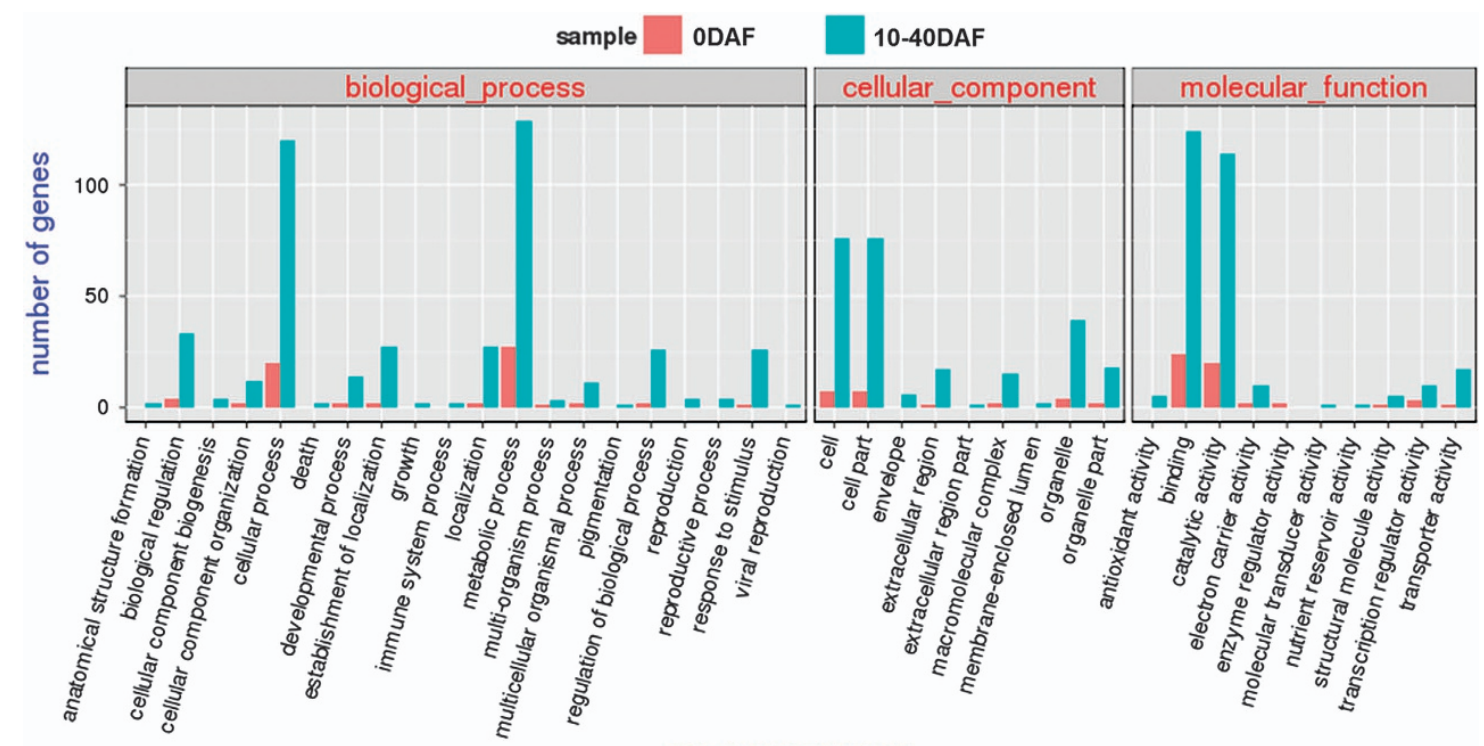

GO ANNOTATION

Figure 2. Functional classification of the genes specifically expressed in 'Flavor No. 4' female flower or fruit flesh within the category of biological process, molecular function and cellular component.

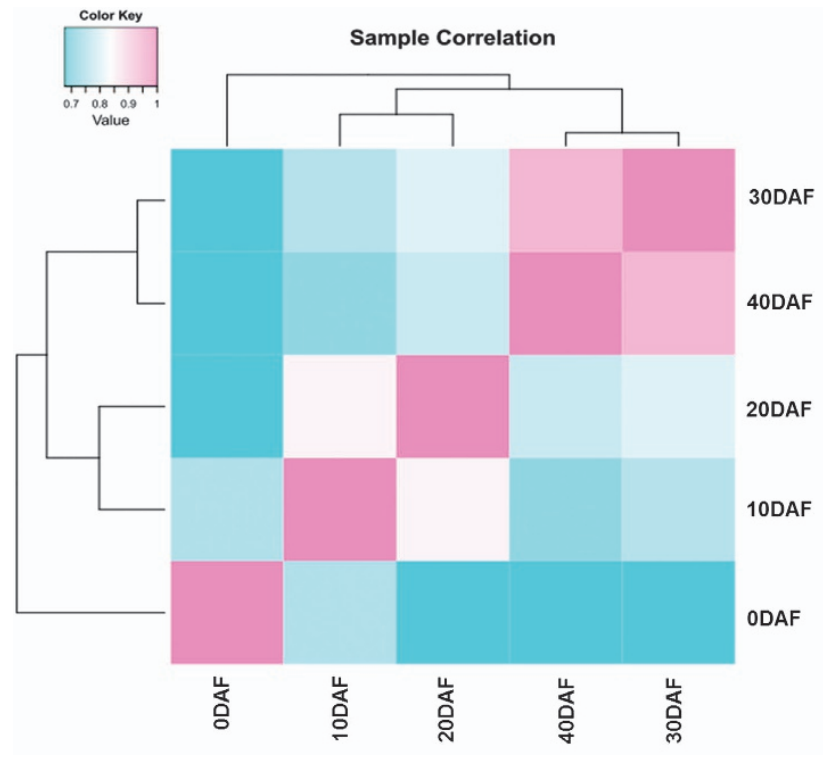

Figure 3. Hierarchical clustering analysis of gene expression levels in each of the five samples $(0,10,20,30$ and 40 days after flowering (DAF)). Shades of blue indicate lowered expression, relative to the mean across samples, whereas shades of pink indicate higher expression relative to the mean.

Cellular process (2189 genes in female flowers, 2289 genes in fruit flesh), metabolism process (2174 genes in female flowers, 2276 genes in fruit flesh) and biological regulation (594 genes in female flowers, 623 genes in fruit flesh) were among the most highly represented groups within the biological process category, indicating both the female flower and fruit flesh tissue was undergoing extensive metabolic activities. In addition, genes involved in other important biological processes such as establishment of localization, regulation of biological process and response to stimulus were also identified. GO terms were also assigned to the specifically expressed genes in female flowers and fruit flesh. As shown in Figure 2, compared with fruit flesh tissues, enzyme regulator activity was represented within the molecular function category of flower tissues. In the fruit flesh, antioxidant, molecular transducer and nutrient reservoir activities within the molecular function category; anatomical structure, cellular component organization, growth, immune system within the biological process; and envelope, extra cellular region and membraneenclosed lumen within the cellular component were the unique groups compared with the flower tissues. This finding suggested that the genes categorized in these groups may exert tissuespecific function during plant development.

Hierarchical clustering analysis was used to investigate the expression patterns of genes in female flowers and fruit flesh. As shown in Figure 3, the 10 DAF was assigned to the adjacent node of $20 \mathrm{DAF}, 30 \mathrm{DAF}$ was grouped into the same cluster of $40 \mathrm{DAF}$, while 0 DAF showed distinct gene expression pattern. This finding demonstrated tissue-specific gene expression of flower different from fruit flesh. During fruit development, similarities in gene expression pattern were observed between 10 and $20 \mathrm{DAF}$, and 30 and $40 \mathrm{DAF}$, suggesting that the gene expression was significantly altered from 20-30 DAF, with implications for fruit development.

DEGs during fruit development

A total of 7892 genes showed significant upregulation or downregulation ( $P$-value $<0.01$, foldchange $>2$ or $<0.5)$ during fruit development.

On the basis of similar kinetic patterns of expression, all 7892 DEGs were classified into 15 types of clusters (Figure 4). The gene expression patterns of cluster 1 (259 DEGs), cluster 6 (233 DEGs), cluster 9 (204 DEGs) and cluster 11 (263 DEGs) exhibited similar changes during fruit development. The gene expression levels showed a rapid increase from 20 to $30 \mathrm{DAF}$, but changed little between 10 and 20 DAF and between 30 and 40 DAF. Genes in this group may function in the middle stages of fruit development. The gene expression patterns of cluster 4 (207 DEGs), cluster 13 (19 DEGs), cluster 14 (188 DEGs) and cluster 15 (107 DEGs) were similar. The gene expression levels showed rapid decrease from 20 to $30 \mathrm{DAF}$, but changed little during the rest of the developmental stages. Genes in this group may also have a role in the middle stages of fruit development. The gene expression patterns of cluster 3 (288 DEGs), cluster 8 (195 DEGs) and cluster 12 (208 

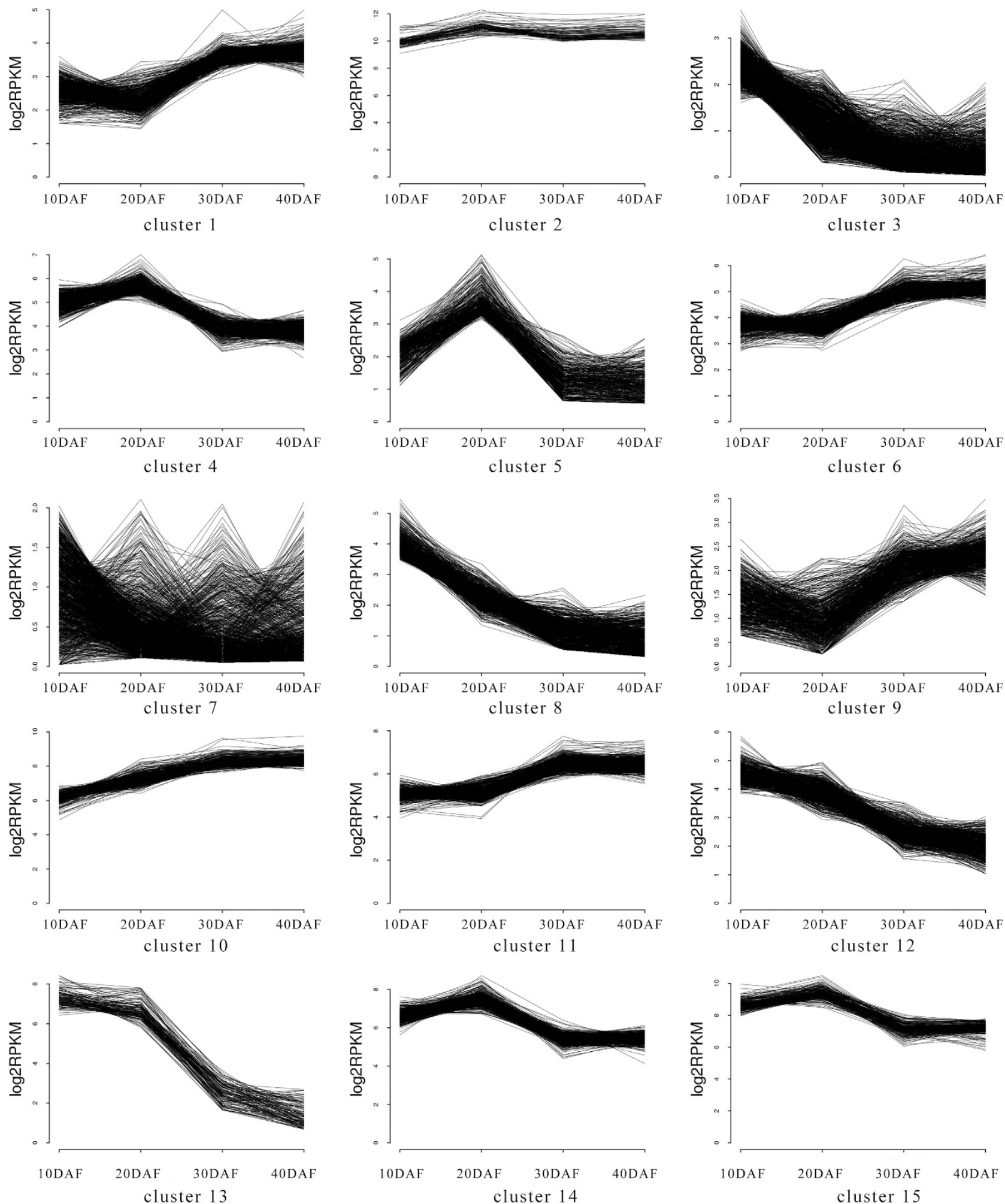

Figure 4. Gene expression pattern obtained by $K$-means clustering. Differentially expressed genes (DEGs) in fruit flesh were categorized into 15 clusters depending on their expression during fruit development. Levels of gene expression were represented along the $y$ axis as log 2 RPKM, and the stages of fruit development were represented along the $x$ axis as 10, 20,30 and 40 days after flowering (DAF).

DEGs) displayed a continuous decline during fruit development. These genes may have an important role at the young fruit stage. The expression levels of genes in cluster 2 (37 DEGs) and cluster 10 (192 DEGs) were maintained at a high level during the entire development. The gene expression levels in cluster 2 were altered minimally at different development stages. The gene expression levels in cluster 10 showed a slight increase during fruit development. Genes in these two clusters may exert a 
6

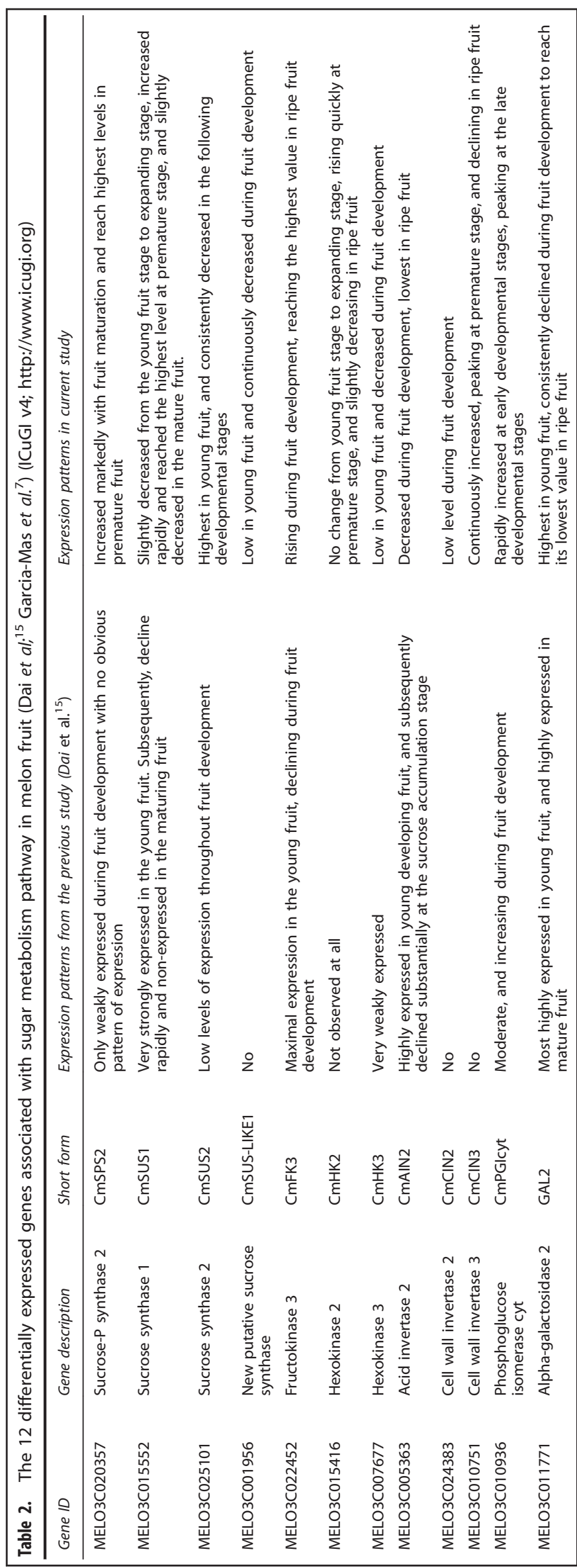

fundamental role in the fruit developmental process. Genes in cluster 5 (106 DEGs) were specifically highly expressed at 20 DAF, indicating a close relationship with fruit expansion. Genes in cluster 7 (394 DEGs) had a relatively low expression in the fruit developmental process.

Analysis of the candidate genes involved in fruit development and ripening

To systematically assess the biological functions of the candidate genes, KEGG enrichment analysis was performed. The candidate DEGs were assigned to 16 KEGG pathway $(P$-value $\leqslant 0.05)$ with significant enrichment.

Genes mediating sugar metabolism

Eighty-three DEGs were found to be associated with sucrose metabolism, belonging to 13 different gene expression clusters, of which, 12 genes were associated with sugar metabolism in melon fruit $^{7,15}$ (ICuGl v4; http://www.icugi.org). In the current study, the sucrose phosphate synthase 2 (CMSPS2) (MELO3C020357) increased markedly with fruit maturation and reached the highest levels in premature fruit. Three sucrose synthases (CMSUS1, CmSUS2 and CmSUS-LIKE1) (MELO3C015552, MELO3C025101 and MELO3C001956) showed different expression patterns during fruit development and ripening. The expression level of CmSUS1 was slightly decreased from the young fruit stage to expanding stage, and then increased rapidly to the highest level at premature stage, with a slight decrease in the mature fruit. The expression level of CmSUS2 was the highest in young fruit, and consistently decreased in the following developmental stages. The expression level of CMSUS-LIKE1 was relatively low in young fruits and was continuously decreased during fruit development. Two hexokinases (CmHK2 and CmHK3) (MELO3C015416 and MELO3C007677) were differentially expressed during fruit development of Hami melon. The expression level of CmHK2 was similar in the young fruit and expanding stages, increased rapidly at premature stage, and slightly decreased in the ripe fruit. The CmHK3 expression was low in young fruit and decreased during fruit development. The expression level of fructokinase 3 (CmFK3) (MELO3C022452) gradually increased during fruit development, reaching the highest value in the ripe fruit. The expression level of acid invertases 2 (CmAIN2) (MELO3C005363) showed the opposite trend and declined during fruit development. Two cell wall invertases (CmCIN2 and CmCIN3) (MELO3C024383 and MELO3C010751) were differentially expressed during fruit development. The expression level of $\mathrm{CmCIN3}$ was continuously increased, peaking in the premature stage, and declined in the ripe fruit. The expression of $\mathrm{CmCIN2}$ was low during fruit development. The expression level of phosphoglucose isomerase cyt (CmPGlcyt) (MELO3C010936) was rapidly increased in the early developmental stages, peaking at the late developmental stages. The expression level of alpha-galactosidase 2 (GAL2) (MELO3C011771) was the highest in young fruit, consistently declined during fruit development and reached its lowest value in ripe fruit. Subsequently, we compared the gene expression patterns of these 12 genes in our transcriptome profiles from the previous study ${ }^{14}$ (Table 2), most of which showed varying trends.

In addition to the above 12 DEGs, 71 DEGs were also found associated with sugar metabolism pathways during Hami melon fruit development and ripening. Their expression patterns were categorized into 13 clusters.

Genes involved in citrate acid cycle

The 13 DEGs identified in this study were possibly related to CA cycle during Hami melon fruit development, and were categorized into 9 expression pattern clusters (Table 3). Three phosphoenolpyruvate carboxykinases (PCK1, PCK2 and PCK3) (MELO3C007687, MELO3C018994 and MELO3C003491) were differentially 


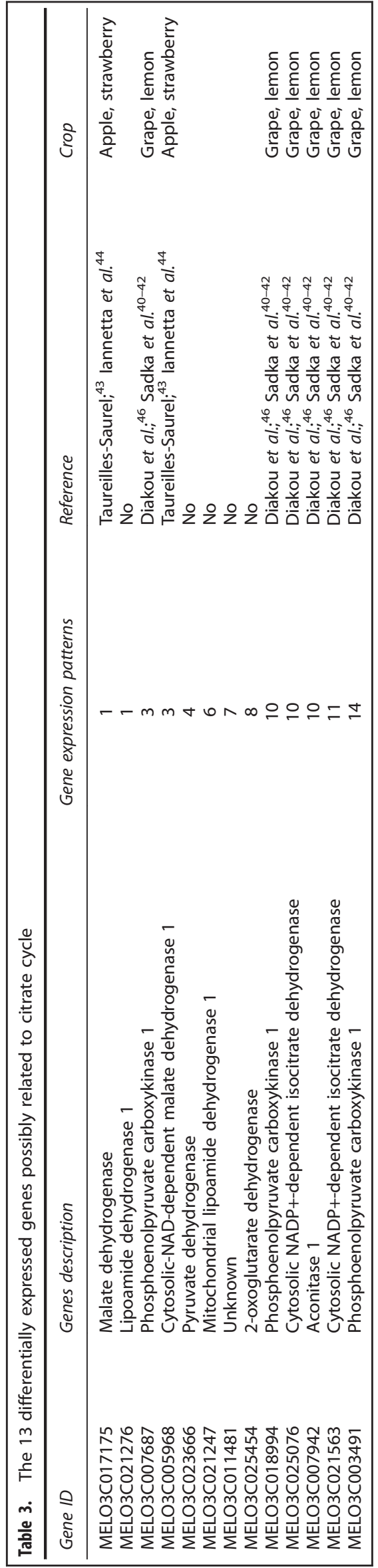

expressed during fruit development. The expression level of PCK1 was the highest in the young fruit, drastically declined to the lowest level in ripe fruit. The expression level of $P C K 2$, on the contrary, slightly increased in the early and middle stages of fruit development, and then drastically increased at the late stages of fruit development. The expression level of $P C K 3$ remained the same in young fruit and expanding stages, and then rapidly decreased in the middle developmental stage, reaching a low at premature and mature stages. Two cytosolic NADP+-dependent isocitrate dehydrogenases ( $c / C D H 1$ and $C I C D H 2$ ) (MELO3C005968 and MELO3C021563) were differentially expressed during fruit development. The $\mathrm{CICDH} 2$ expression level increased markedly in the middle of fruit development, reached the highest level in premature fruit and slightly decreased in the mature fruit, whereas the expression of $\mathrm{ClCDH} 1$ was maintained at a low level throughout the fruit development process. Two lipoamide dehydrogenase 1 (LPD1) (MELO3C021276 and MELO3C021247) genes showed similar changes during fruit development, with increased expression levels during fruit development and the highest value in ripe fruit. Two malate dehydrogenase (MD1, MD2) (MELO3C017175, MELO3C005968) genes showed the opposite trend. Although the expression level of MD1 increased, the expression level of MD2 decreased during fruit development. The expression level of aconitate 1 (ACO1) (MELO3C007942) was markedly increased in the middle stage of fruit development, and reached the highest value in ripe fruit. The expression level of 2-oxoglutarate dehydrogenase $(\mathrm{OGDH})(\mathrm{MELO} 3 \mathrm{C025454)}$ was the highest in young ripe fruit, and drastically declined in the expanding stage and reached the lowest value in ripe fruit. Pyruvate dehydrogenase (PD) (MELO3C023666) highly expressed in young fruit, declined to the lowest level in premature fruit and slightly increased in ripe fruit.

Verification of DEGs during Flavor No. 4 Hami melon fruit development

In order to verify the expression patterns of DEGs involved in fruit development, eight genes were randomly selected for quantitative real-time PCR analysis. Three genes ( $A C O 1, C S Y 3$ and SDH1-1) were involved in citrate cycle, two genes (SUS5 and UGT73C2) in carbohydrate metabolism, and three were transcription factors (AP2, ANAC100 and SPL13). As shown in Figure 5, although the exact gene expression pattern of the selected genes at several time points varied between RNA-seq and quantitative real-time PCR analysis, their altered expression was similar. These results confirmed the accuracy of our transcriptome profiling.

\section{DISCUSSION}

The RNA-seq study detected 23299 genes expressed in female flowers and fruit flesh during 'Flavor No. 4' Hami melon development. Of these genes, 554 were specifically expressed in female opening flowers and 3260 were specifically expressed in fruit flesh tissues. These genes were assigned to various functional categories and may exert tissue-specific function during plant development. Hierarchical clustering analysis revealed a fundamental change in gene expression pattern between expanding and premature stages, with significant role in fruit development and ripening. A total of 7892 DEGs were identified during fruit development, and categorized into 15 different expression clusters. The candidate DEGs were assigned to 16 KEGG pathways with significant enrichment.

Hierarchical clustering analysis revealed a fundamental change in gene expression patterns between expanding and premature stages. Among the 7892 DEGs, 259 DEGs in cluster 1, 233 DEGs in cluster 6, 204 DEGs in cluster 9 and 263 DEGs in cluster 11 showed a rapid increase in expression from expanding to premature stages, with little change during the rest of development stages. 

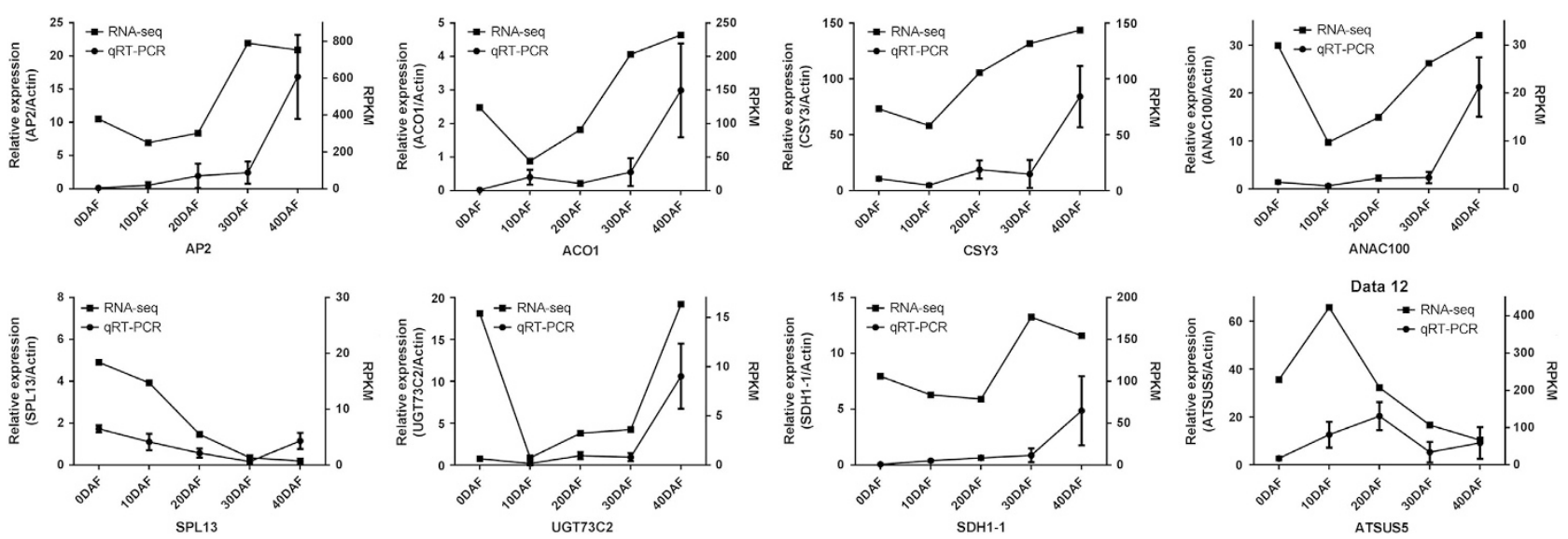

Figure 5. Quantitative real-time PCR (qRT-PCR) analysis of DEGs related to fruit development. The left $y$ axis represents the relative gene expression levels analyzed by qRT-PCR and the right $y$ axis represents the RPKM value analyzed by RNA sequencing (RNA-seq). Bars represent the s.e. $(n=3)$.

The 207 DEGs in cluster 4, 19 DEGs in cluster 13, 188 DEGs in cluster 14 and 107 DEGs in cluster 15 showed a rapid decrease in expression from expanding to premature stage. In addition, 106 DEGs in cluster 5 were highly expressed in the expanding stage. All these DEGs probably had a critical role in Hami melon fruit development.

In the KEGG enrichment analysis, the candidate DEGs associated with fruit development were assigned to 16 functional pathways with significant enrichment. Of these, the plant hormone signal transduction pathway mediated fruit ripening. ${ }^{16}$ In this pathway, the ethylene and fruit-specific transcription factors together with epigenome modification mediated the transition of fruit to the ripening stage. ${ }^{17}$ In this study, DEGs in plant hormone signal transduction pathway showed different gene expression patterns during the fruit development process, suggesting a role in the regulation of fruit development.

Melons are consumed for their sweet fruit, ${ }^{18}$ characterized by the accumulation of sucrose during the late stage of fruit development. ${ }^{19-23}$ In this study, the sucrose content increased markedly from fruit expansion to mature stage, and reached its highest level in ripe fruit, demonstrating the rapid accumulation of sucrose in the medium and late developmental stages in 'Flavor No. 4' Hami melon fruit. Sucrose accumulation in the melon fruit mesocarp is a developmentally regulated process related to metabolic transition. ${ }^{20,24-31}$ The detailed sucrose metabolic pathway in melon has been introduced in a previous study. ${ }^{15}$ In the present study, 83 DEGs were found to mediate sugar metabolism. Of these, 12 genes have been related to sucrose accumulation in melon fruit. ${ }^{7,15}$ We compared the expression pattern of the 12 genes in this study with the transcriptome profiles in a previous study. ${ }^{15}$ Our results showed three genes, Hexokinase3, CmAIN2 and CmPGlcyt, with similar alterations during fruit development, and five genes, CmSPS2, CmSUS2, CmFK3, Hexokinase 2 and GAL2, showing opposite trends, while the expression of CMSUS-LIKE1, CmCIN2 and CmCIN3 was not detected in Dai's study (Table 3). Different melon varieties and growing conditions led to differences in gene expression pattern in different studies.

In sweet melons, sucrose is the primary component determining fruit quality. ${ }^{18,19}$ However, a few varieties accumulate high levels of acid in melon fruit. ${ }^{32-35}$ Breeders combine the high acidity and high sugar traits of melons to develop dessert cultivars. $^{36}$ 'Flavor No. 4' Hami melon is one such cultivar with unique sweet and sour taste, with CA as the major organic acid in its ripe fruit. ${ }^{37}$ In this study, the CA content in fruit flesh showed a rapid increase from expanding to mature stages, indicating the $C A$ accumulation in the late developmental stage. Generally, the acid content of a fruit is determined by the balance of acid synthesis and degradation. Organic acid metabolism in fruits is a complex physiological process. As described before, ${ }^{36}$ PEPC catalyzes the synthesis of oxaloacetic acid (OAA) from PEP, MD catalyzes malic acid synthesis from OAA, both of which enter the tricarboxylic acid cycle. Citrate synthase (CS) catalyzes CA synthesis using acetyl coenzyme A (Ac-CoA) and OAA. CA enters the cytoplasm, and accumulates in the vacuoles with the help of $\mathrm{H}^{+}$pump active transport carrier. As the CA level in vacuoles accumulates to a high level, it is released into the cytoplasm. In the cytoplasm, cytaconitase $(A C O)$ converts citrate to isocitrate, which is unstable, and is converted by isocitrate dehydrogenase (NADP-IDH) to generate ketone glutaric acid. In different fruit species, different enzymes catalyze organic acid metabolism. Phosphoenolpyruvate carboxylase $(P C K), C S$, aconitase $(A C O)$ and isocitrate dehydrogenase $(c / C D H)$ in grape, ${ }^{38}$ pineapple, ${ }^{39}$ peach $^{38}$ and lemon, ${ }^{40-42}$ respectively, and $M D$ in apple, strawberry and grape ${ }^{43,44}$ have a critical role in the accumulation of organic acids in fruits. In the present study, we found that 13 DEGs possibly mediated CA accumulation. The $P C K$ and $M D$ were involved upstream of $C A$ synthesis. PCK is the key enzyme that catalyzed the conversion of PEP to OAA, and MD catalyzed conversion of OAA to malic acid, which entered the CA cycle. In this study, the expression level of $P C K 1$ was the highest in young fruit, drastically declined to its lowest level in ripe fruit. The expression level of $P C K 2$ was slightly increased in the early and middle stages of fruit development, drastically increased at the late stage of fruit development. The $P C K 3$ expression was rapidly decreased in the middle stage, to reach a low level at premature and mature stages. The MD1 expression level increased during the fruit development, while the MD2 level decreased during fruit development. The rapid increase in $P C K 2$ and $M D 1$ may contribute to $C A$ accumulation in mature fruit. Aconitase 1 (ACO1) and cytosolic NADP ${ }^{+}$-dependent isocitrate dehydrogenase $(C / C D H)$ mediate downstream CA synthesis. ACO1 has an important role in catalyzing isocitrate formation, resulting in CA degradation. ${ }^{45}$ In our study, the expression level of aconitate 1 (ACO1) (MELO3C007942) was markedly increased in the middle stage of fruit development, and reached the highest value in ripe fruit. The $\triangle I C D H 2$ increased markedly in the middle stage of fruit development, reaching the highest level in premature fruit and slightly decreased in the mature fruit, whereas the expression of $C I C D H 1$ was maintained at a low level throughout the fruit development process. The upstream enzymes of citrate acid cycle work synergistically to accumulate CA. Excessive cellular levels of CA activate the downstream enzymes, which initiate its degradation. 
We identified the DEGs mediating various pathways in fruit development and ripening of 'Flavor No. 4'. Our results provide valuable insights in elucidating the molecular mechanism of sucrose and CA accumulation during melon fruit development.

\section{CONFLICT OF INTEREST}

The authors declare no conflict of interest.

\section{ACKNOWLEDGEMENTS}

We thank ABlife, Inc., (Wuhan, China) for technical assistance, data analysis and manuscript writing support. This study was supported by the National Natural Science Foundation of China (31160395, http://www.nsfc.gov.cn), Postdoctoral Research Project (131620), Special Fund for Agro-scientific Research in the Public Interest of Ministry of Agriculture of the People's Republic of China (201203004).

\section{REFERENCES}

1 Erickson DL, Smith BD, Clarke AC, Sandweiss DH, Tuross N. An Asian origin for a 10,000-year-old domesticated plant in the Americas. Proc Natl Acad Sci USA 2005; 102: 18315-18320.

2 Pech J-C, Bouzayen M, Latché A. Climacteric fruit ripening: ethylene-dependent and independent regulation of ripening pathways in melon fruit. Plant Sci 2008; 175: 114-120.

3 Boualem A, Fergany M, Fernandez R, Troadec C, Martin A, Morin H et al. A conserved mutation in an ethylene biosynthesis enzyme leads to andromonoecy in melons. Science 2008; 321: 836-838

4 Martin A, Troadec C, Boualem A, Rajab M, Fernandez R, Morin H et al. A transposon-induced epigenetic change leads to sex determination in melon. Nature 2009; 461: 1135-1138.

5 Zhang B, Tolstikov V, Turnbull C, Hicks LM, Fiehn O. Divergent metabolome and proteome suggest functional independence of dual phloem transport systems in cucurbits. Proc Natl Acad Sci USA 2010; 107: 13532-13537.

6 Dahmani-Mardas F, Troadec C, Boualem A, Lévêque S, Alsadon AA, Aldoss AA et al. Engineering melon plants with improved fruit shelf life using the TILLING approach. PLoS One 2010; 5: e15776.

7 Garcia-Mas J, Benjak A, Sanseverino W, Bourgeois M, Mir G, González VM et al. The genome of melon (Cucumis melo L.). Proc Natl Acad Sci USA 2012; 109: 11872-11877.

8 Corbacho J, Romojaro F, Pech J-C, Latché A, Gomez-Jimenez MC. Transcriptomic events involved in melon mature-fruit abscission comprise the sequential induction of cell-wall degrading genes coupled to a stimulation of endo and exocytosis. PLoS One 2013; 8: e58363.

9 Blanca J, Cañizares J, Ziarsolo P, Nuez F, Picó B. Melon transcriptome characterization. SSRs and SNPs discovery for high throughput genotyping across the species. Plant Genome 2011; 4: 118-131.

10 Obando-Ulloa JM, Eduardo I, Monforte AJ, Fernández-Trujillo JP. Identification of QTLs related to sugar and organic acid composition in melon using nearisogenic lines. Sci Hortic 2009; 121: 425-433.

11 Kim D, Pertea G, Trapnell C, Pimentel H, Kelley R, Salzberg SL. TopHat2: accurate alignment of transcriptomes in the presence of insertions, deletions and gene fusions. Genome Biol 2013; 14: R36.

12 Robinson MD, McCarthy DJ, Smyth GK. edgeR: a Bioconductor package for differential expression analysis of digital gene expression data. Bioinformatics 2010; 26: 139-140.

13 Götz S, Garcia-Gózmez JM, Terol J, Williams TD, Nagaraj SH, Nueda MJ et al. Highthroughput functional annotation and data mining with the Blast2GO suite. Nucleic Acids Res 2008; 36: 3420-3435.

14 Livak KJ, Schmittgen TD. Analysis of relative gene expression data using real-time quantitative PCR and the $2-\Delta \Delta C T$ method. Methods 2001; 25: 402-408.

15 Dai N, Cohen S, Portnoy V, Tzuri G, Harel-Beja R, Pompan-Lotan M et al. Metabolism of soluble sugars in developing melon fruit: a global transcriptional view of the metabolic transition to sucrose accumulation. Plant Mol Biol 2011; 76: 1-18.

16 Vendrell M, Palomer X. Hormonal control of fruit ripening in climacteric fruits. Acta Hortic 1998; 463: 325-334.

17 Zhong S, Fei Z, Chen Y-R et al. Single-base resolution methylomes of tomato fruit development reveal epigenome modifications associated with ripening. Nat Biotechnol 2013; 31: 154-159.

18 Yamaguchi M, Hughes D, Yabumoto K, Jennings W. Quality of cantaloupe muskmelons: variability and attributes. Sci Hortic 1977; 6: 59-70.

19 Burger Y, Schaffer AA. The contribution of sucrose metabolism enzymes to sucrose accumulation in Cucumis melo. J Am Soc Hortic Sci 2007; 132: 704-712.
20 Hubbard NL, Huber SC, Pharr DM. Sucrose phosphate synthase and acid invertase as determinants of sucrose concentration in developing muskmelon (Cucumis melo L.) fruits. Plant Physiol 1989; 91: 1527-1534.

21 Rosa JT Changes in composition during ripening and storage of melons: University of California 1928.

22 Schaffer AA, Aloni B, Fogelman E. Sucrose metabolism and accumulation in developing fruit of Cucumis. Phytochemistry 1987; 26: 1883-1887.

23 Schaffer AA, Pharr DM, Madore MA. Cucurbits. In: Zamski E, Schaffer AA (eds.) Photoassimilate distribution in plants and crops. New York: CRC Press, 1996; 729-757.

24 Lester GE, Arias LS, Gomez-Lim M. Muskmelon fruit soluble acid invertase and sucrose phosphate synthase activity and polypeptide profiles during growth and maturation. J Am Soc Hortic Sci 2001; 126: 33-36.

25 Carmi N, Zhang G, Petreikov M, Gao Z, Eyal Y, Granot D et al. Cloning and functional expression of alkaline a-galactosidase from melon fruit: similarity to plant SIP proteins uncovers a novel family of plant glycosyl hydrolases. Plant $J$ 2003; 33: 97-106.

26 Dai N, Petreikov M, Portnoy V, Katzir N, Pharr DM, Schaffer AA. Cloning and expression analysis of a UDP-galactose/glucose pyrophosphorylase from melon fruit provides evidence for the major metabolic pathway of galactose metabolism in raffinose oligosaccharide metabolizing plants. Plant Physiol 2006; 142: 294-304.

27 Gao Z, Schaffer AA. A novel alkaline a-galactosidase from melon fruit with a substrate preference for raffinose. Plant Physiol 1999; 119: 979-988.

28 Gao Z, Petreikov M, Burger Y, Shen S, Schaffer AA. Stachyose to sucrose metabolism in sweet melon (Cucumis melo) fruit mesocarp during the sucrose accumulation stage. In: Lebeda Paris (ed). Progress in cucurbit genetics and breeding research. Olomouc: Palacký University, 2004; 471-475.

29 Keller F, Pharr DM. Metabolism of carbohydrates in sinks and sources: galactosylsucrose oligosaccharides. In: Zamski E, Schaffer AA (eds.). Photoassimilate Distribution in Plants and Crops: Source-Sink Relationships. New York: CRC Press, 1996; 157-183.

30 Chrost B, Schmitz K. Changes in soluble sugar and activity of a-galactosidases and acid invertase during muskmelon (Cucumis melo L.) fruit development. J Plant Physiol 1997; 151: 41-50.

31 Mitchell DE, Gadus MV, Madore MA. Patterns of assimilate production and translocation in muskmelon (Cucumis melo L.) I. Diurnal patterns. Plant Physiol 1992; 99: 959-965.

32 Cohen S, Tzuri G, Harel-Beja R, Itkin M, Portnoy V, Sa'ar U et al. Co-mapping studies of QTLs for fruit acidity and candidate genes of organic acid metabolism and proton transport in sweet melon (Cucumis melo L.). Theor Appl Genet 2012; 125: 343-353.

33 Burger Y, Paris HS, Cohen R et al. Genetic diversity of Cucumis melo. Hortic Rev 2009; 36: 165-198.

34 Danin-Poleg Y, Tadmor Y, Tzuri G, Reis N, Hirschberg J, Katzir N. Construction of a genetic map of melon with molecular markers and horticultural traits, and localization of genes associated with ZYMV resistance. Euphytica 2002; 125: 373-384.

35 Kubicki B. Inheritance of some characters in muskmelons (Cucumis melo). Genet Polonica 1962; 3: 265-274.

36 Burger Y, Sa'ar U, Distelfeld A, Katzir N, Tadmor Y, Lewinsohn E et al. Development of sweet melon (Cucumis melo) genotypes combining high sucrose and organic acid content. J Am Soc Hortic Sci 2003; 128: 537-540.

37 Tang M, Bie Z-I, Wu M-Z, Yi H-p, Feng J-x. Changes in organic acids and acid metabolism enzymes in melon fruit during development. Sci Hortic 2010; 123: 360-365.

38 Moing A, Rothan C, Svanella L et al. Role of phosphoenolpyruvate carboxylase in organic acid accumulation during peach fruit development. Physiol Plantarum 2000; 108: 1-10.

39 Saradhuldhat $\mathrm{P}$, Paull RE. Pineapple organic acid metabolism and accumulation during fruit development. Sci Hortic 2007; 112: 297-303.

40 Sadka A, Dahan E, Cohen L, Marsh KB. Aconitase activity and expression during the development of lemon fruit. Physiol Plantarum 2000; 108: 255-262.

41 Sadka A, Dahan E, Or E, Cohen L. NADP+-isocitrate dehydrogenase gene expression and isozyme activity during citrus fruit development. Plant Sci 2000; 158: $173-181$.

42 Sadka A, Dahan E, Or E, Roose ML, Marsh KB, Cohen L. Comparative analysis of mitochondrial citrate synthase gene structure, transcript level and enzymatic activity in acidless and acid-containing Citrus varieties. Funct Plant Biol 2001; 28: 383-390.

43 Taureilles-Saurel C, Romieu CG, Robin J-P, Flanzy C. Grape (Vitis vinifera L.) malate dehydrogenase. II. Characterization of the major mitochondrial and cytosolic isoforms and their role in ripening. Am J Enol Viticult 1995; 46: 29-36.

44 lannetta PP, Escobar NM, Ross HA, Souleyre EJ, Hancock RD, Witte CP et al. Identification, cloning and expression analysis of strawberry (Fragaria $\times$ ananassa) mitochondrial citrate synthase and mitochondrial malate dehydrogenase. Physiol Plantarum 2004; 121: 15-26. 
45 Wang X-Y, Wang P, Qi Y-P, Zhou CP, Yang LT, Liao XY et al. Effects of granulation on organic acid metabolism and its relation to mineral elements in Citrus grandis juice sacs. Food Chem 2014; 145: 984-990.

46 Diakou P, Svanella L, Raymond P, Gaudillere JP, Moing A. Phosphoenolpyruvate carboxylase during grape berry development: protein level, enzyme activity and regulation. Australian Journal of Plant Physiology 2000; 27: 221-229. (i) This work is licensed under a Creative Commons Attribution 4.0 International License. The images or other third party material in this article are included in the article's Creative Commons license, unless indicated otherwise in the credit line; if the material is not included under the Creative Commons license, users will need to obtain permission from the license holder to reproduce the material. To view a copy of this license, visit http://creativecommons.org/licenses/ by/4.0/ 